\title{
Field engineering by continuous hole injection in silicon detectors irradiated with neutrons
}

\author{
G. Kramberger*, V. Cindro, I. Mandić, M. Mikuž, M. Zavrtanik \\ Department of Physics, Jožef Stefan Institute, University of Ljubljana, SI-1000 Ljubljana, Slovenia
}

Received 28 May 2002; received in revised form 17 October 2002; accepted 29 October 2002

\begin{abstract}
The electric field in irradiated silicon diodes was modified by manipulating the occupation of deep levels. This was achieved by continuous injection of holes using light illumination. Effective trapping probabilities and space-charge concentrations were measured in this operating mode. The bias voltage needed to establish the electric field in the whole detector volume, charge collection, power consumption and shot noise were investigated. The optimum operation point was found to be independent of irradiation fluence, providing a robust way of operating highly irradiated detectors, even if irradiated in a non-uniform way.
\end{abstract}

(C) 2002 Elsevier Science B.V. All rights reserved.

PACS: 85.30.De; 29.40.Wk; 29.40.Gx

Keywords: Continuous carrier injection; Effective space charge; Effective carrier trapping time; Silicon detectors; Charge collection efficiency

\section{Introduction}

The exposure of silicon detectors to high levels of radiation, such as in the LHC experiments, or higher, deteriorates their performance. The degradation is caused by the creation of defects which appear as a consequence of Si atoms being knocked out of their original positions by high-energy particles (see for example [1]). The most important deteriorating effect of this damage is the increase of

\footnotetext{
*Corresponding author. Current address: DESY, Notkestrasse 85, D-22607 Hamburg, Germany. Tel.: + 49-40-89984728 .

E-mail address: gregor.kramberger@desy.de (G. Kramberger).
}

reverse-bias voltage which must be applied to obtain an efficient detector. The maximum applicable operation voltage, restricted by the potential danger of electric breakdown and increased power consumption, becomes the limiting factor at high fluences. Oxygen enrichment of FZ-silicon reduces this problem if the damage is caused by charged hadrons; however, this improvement is absent after neutron irradiation $[2,3]$. Other ways to deal with the problem of a high operational voltage are the use of detectors whose radiation hard properties originate from their geometric properties (thin detectors [4], 3D detectors [5]) or operation of detectors at cryogenic temperatures, where the effective space charge is reduced. This phenomenon is known as the Lazarus effect [6]. 
It has been shown that the injection of carriers into the depleted region of an irradiated detector, either by illumination of the surface by pulsed visible light $[7,8]$ or through electron-hole pair creation in the detector bulk by an infrared laser, which simulates a minimum ionizing particle (MIP) [9], can change the electric field in the detector by trapping of non-equilibrium carriers in radiation-induced deep levels. In this work we investigated space-charge manipulation by injection of charge carriers using continuous illumination at close to room temperatures.

By continuous (DC) illumination of one detector side with light of short wavelength, free the electron or hole concentration in the active volume of the detector is enhanced, influencing the occupation probability of traps contributing to the space charge. At temperatures above $T \approx$ $-140^{\circ} \mathrm{C}$, the bulk of a heavily irradiated silicon detector is effectively p-type, and thus the space charge is negative $[10,11]$. With continuous hole injection, the space charge can be modified through the balance between trapping and emission of the drifting holes. The modification of the space charge can reduce the voltage needed to establish the electric field throughout the whole detector thickness. Operating detectors in the presence of continuous carrier injection had already been proposed in Refs. [12,13], but experimentally explored mostly at low temperatures $\left(T<-120^{\circ} \mathrm{C}\right)$ [14-16]. The first experimental demonstration of this operation mode at higher temperatures $\left(T>-10^{\circ} \mathrm{C}\right)$ was published in Ref. [17]. In the following sections, we present a more detailed and quantitative study of TCT signals in irradiated silicon diodes in the presence of continuous carrier injection, as well as an assessment of the practical aspects of detector operation in such an operational mode.

\section{Measurement set-up and samples}

The transient current technique (TCT) set-up described in detail in Ref. [17] was used to measure the response of detectors to short light pulses. Silicon diodes were mounted in a temperaturecontrolled liquid-nitrogen cryostat with optical
Table 1

Irradiation fluences and depletion voltages measured by the $C-V$ method; $v=10 \mathrm{kHz}$ at $T=20^{\circ} \mathrm{C}$

\begin{tabular}{lc}
\hline Fluences $\left(10^{13} \mathrm{n} / \mathrm{cm}^{2}\right)$ & $V_{\mathrm{FD}}$ in minimum $(\mathrm{V})$ \\
\hline 5 & 70 \\
7.5 & 120 \\
10 & 160 \\
20 & 290 \\
\hline
\end{tabular}

Diodes were processed by ST Microelectronics on $300 \mu \mathrm{m}$ thick silicon wafers from Wacker with an initial resistivity of $15 \mathrm{k} \Omega \mathrm{cm}\left(V_{\mathrm{FD}}=10-15 \mathrm{~V}\right)$. The silicon crystals were cut along the $\left\langle\begin{array}{llll}1 & 1 & 1\end{array}\right\rangle$ plane.

windows, allowing light injection from both $\mathrm{p}^{+}$ and $\mathrm{n}^{+}$sides of the diode. Current signals were amplified with a wide-band current amplifier and transmitted to a $500-\mathrm{MHz}$ digitizing oscilloscope. A red laser with a wavelength of $670 \mathrm{~nm}$ (absorption depth in silicon is $3.3 \mu \mathrm{m}$ at $20^{\circ} \mathrm{C}$ ) and pulse width of approximately $1 \mathrm{~ns}$ was used. Continuous light illumination of diodes was made with red light from an $\mathrm{He}-\mathrm{Ne}$ laser $(\lambda=633 \mathrm{~nm})$. The short penetration depth of red light gives the possibility to inject electrons by $\mathrm{p}^{+}$-side illumination and holes by $\mathrm{n}^{+}$-side illumination of a reverse-biased diode.

Samples irradiated to different fluences with neutrons in the TRIGA nuclear reactor of the Jožef Stefan Institute in Ljubljana [18] are listed in Table 1. The listed fluences are scaled to $1 \mathrm{MeV}$ neutron equivalent fluence using hardness factor $\kappa=0.9$ [19]. After irradiation the samples were annealed to the minimum in full depletion voltage ( $\left.V_{\mathrm{FD}}\right)$ and then stored at $T=-17^{\circ} \mathrm{C}$.

\section{Results}

\subsection{Concentration of free holes}

Illumination of a biased diode on the $\mathrm{n}^{+}$side with DC light results in an increase of the hole current flowing through it. From the equation for current density $j=e_{0} p v_{\mathrm{dr}_{\mathrm{h}}}$, it follows

$$
\Delta p=\frac{\Delta I}{e_{0} S v_{\mathrm{dr}_{\mathrm{h}}}}=\frac{\Delta I}{e_{0} S \mu_{\mathrm{h}} E}
$$


where $p$ is the free hole concentration, $\Delta I$ the additional current due to continuous hole injection, $S$ the size of the illuminated area, $v_{\mathrm{dr}_{\mathrm{h}}}$ the drift velocity, $\mu_{\mathrm{h}}$ the mobility of holes, and $E$ the electric field. The reverse-bias current density was in all cases small compared to $\Delta I / S$ and thus neglected, yielding $p \approx \Delta p$ and $n \approx 0$.

The excess concentration of free carriers (Eq. (1)) is governed by two terms: $\Delta I / S$, which is a function of light intensity, and drift velocity $v_{\mathrm{dr}_{\mathrm{h}}}=\mu_{\mathrm{h}}(E) E$, which is a function of bias voltage and to a lesser extent of temperature.

In this work, an average drift velocity was used to calculate the free hole concentration. For a fully from the TCT signal as $\overline{v_{\mathrm{dr}_{\mathrm{h}}}}=D / t_{\mathrm{c}}$ with $D$ being the detector thickness and $t_{\mathrm{c}}$ the charge collection time, defined here as the time between the $50 \%$ points on the rise and fall edges of the current

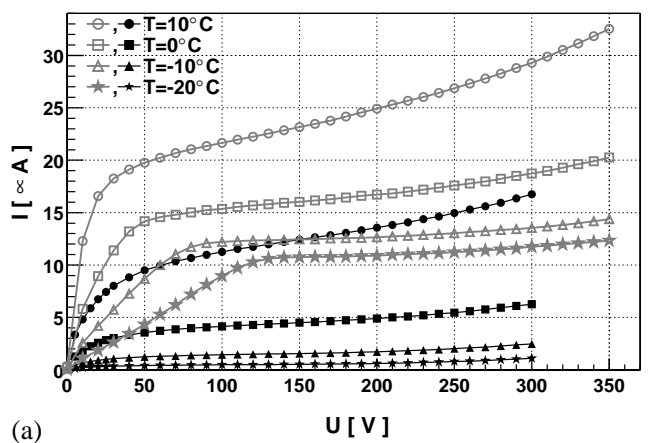
depleted detector, this velocity can be determined

pulse. Hence, the drift velocity is averaged over the detector thickness and thus an average free hole concentration is obtained. This represents an adequate approximation whenever the bias voltage is well above $V_{\mathrm{FD}}$. For example, $\overline{\mathrm{vdr}_{\mathrm{h}}}$ is $1.63 \times 10^{6}$, $2.36 \times 10^{6}$ and $3.57 \times 10^{6} \mathrm{~cm} / \mathrm{s}$ at 90,150 and $300 \mathrm{~V}$ for the data presented in Fig. 2a. The corresponding calculated values using $\overline{v_{\mathrm{dr}_{\mathrm{h}}}}=$ $\mu_{\mathrm{h}}(\bar{E}) \bar{E}$ are $1.7 \times 10^{6}, 2.5 \times 10^{6}$ and $3.85 \times$ $10^{6} \mathrm{~cm} / \mathrm{s}$ if the mobility parametrization taken from [20] is used and $1.5 \times 10^{6}, 2.23 \times 10^{6}$ and $3.51 \times 10^{6} \mathrm{~cm} / \mathrm{s}$ for the mobility parametrization found in [21].

In Fig. 1a, the diode current with and without DC illumination at different temperatures and voltages is shown. It should be noted that less than $10 \%$ of the total diode surface was illuminated, so that the reverse-bias current density is indeed negligible in the illuminated region. It is evident
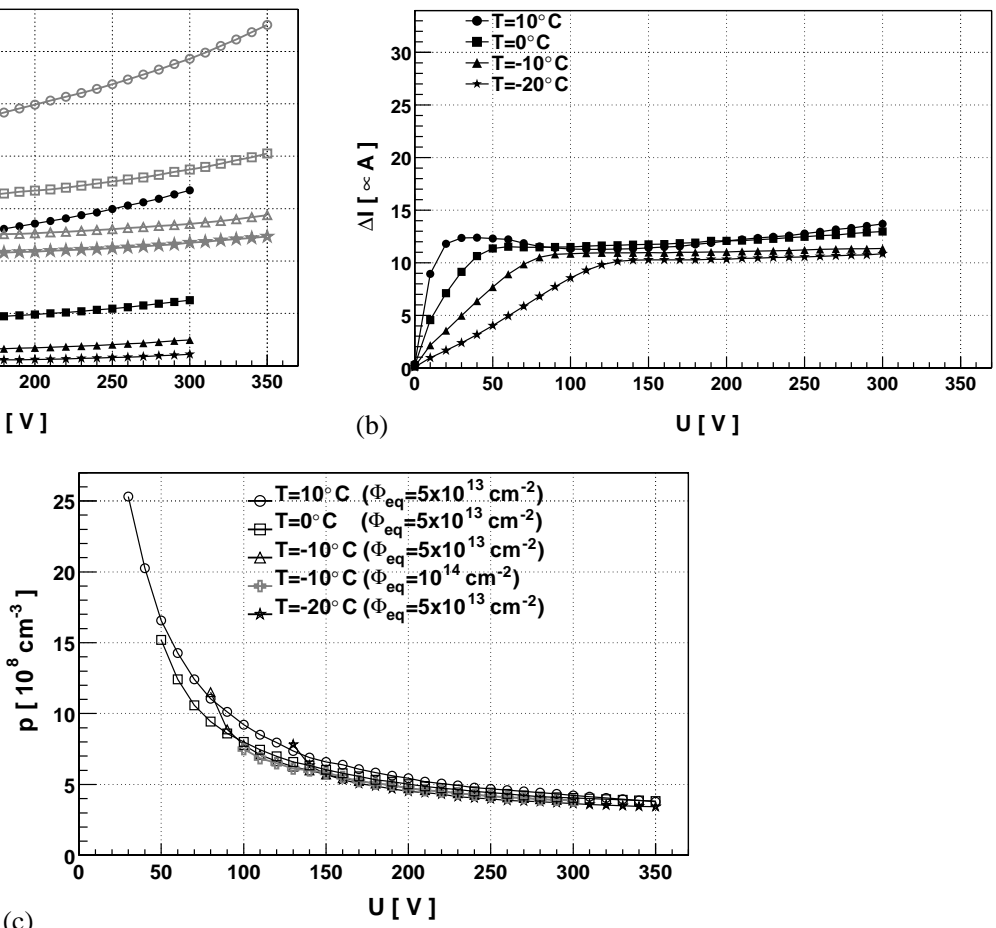

Fig. 1. (a) Diode current with (empty markers) and without (full markers) DC light illumination of the $\mathrm{n}^{+}$side as a function of bias voltage at different temperatures. An approximately constant surplus current due to the DC illumination can be seen in (b). The diode was irradiated to $\Phi_{\mathrm{eq}}=5 \times 10^{13} \mathrm{n} / \mathrm{cm}^{2}$. (c) The hole concentration as a function of bias voltage, calculated from measured $\Delta I$ and charge collection time, at different temperatures for diodes irradiated to different fluences. The DC light intensity was constant so that $\Delta I / S \approx 220 \mu \mathrm{A} / \mathrm{cm}^{2}$. 
that the additional current caused by DC illumination does not depend on temperature or bias voltage for voltages above $V_{\mathrm{FD}}$ (Fig. 1b). In Fig. 1c, the free hole concentrations calculated from the measured $\Delta I / S$ and charge collection time $t_{\mathrm{c}}$ as a function of bias voltage are shown.

\subsection{Space-charge sign inversion with continuous hole injection}

In irradiated detectors defects can act as traps for drifting charge. The trapped charge contributes to the space charge and thus changes the electric field in the detector. In $\mathrm{p}^{+}-\mathrm{n}-\mathrm{n}^{+}$detectors irradiated beyond the inversion point, continuous injection of holes reduces the voltage needed to establish the electric field in the whole detector $\left(V_{\mathrm{FD}}\right)$, whereas continuous injection of electrons increases it [17]. If the concentration of injected holes is high enough, a re-inversion of the spacecharge sign from negative back to positive can occur in an irradiated detector.

Induced hole current pulse shapes acquired in the presence of continuous hole injection show a clear change in slope as the bias voltage is increased (see Fig. 2a). As follows from Eq. (1) the concentration of free holes in the bulk varies with voltage if the intensity of DC light is kept constant. The diode bulk is effectively of n-type (re-inverted) at low voltages and the space charge becomes more p-type (or less positive) as the voltage increases and so the concentration of free holes $(p)$ decreases. But it is important to keep in mind that in Fig. 2a the signal shapes are not corrected for charge trapping so that a change of sign in the signal slope does not directly imply a change in the space-charge sign. In this particular case, the free hole concentration ranged from 3 to $9 \times 10^{8} \mathrm{~cm}^{-3}$ and the space charge was positive for all voltages exhibited in Fig. 2a, as will be demonstrated later.

The effective space-charge concentration and its sign are also strongly dependent on the temperature. The effect of temperature can be seen in Fig. 2b. The effective space charge $\left(N_{\text {eff }}\right)$, at a given voltage and free hole concentration, changes sign as the temperature increases. At $T=-28^{\circ} \mathrm{C}$ the bulk is effectively n-type (re-inverted) at this voltage and light intensity. As the temperature increases emission times decrease, thus a lower number of trapped holes contribute to the space charge which turns more negative. At $T=20^{\circ} \mathrm{C}$ the bulk can be shown to have remained effectively p-type (inverted), as if operated without light illumination.

\subsection{Effective trapping times}

The amount of drifting charge $Q_{\mathrm{e}, \mathrm{h}}$ decreases exponentially with time in an irradiated detector:

$Q_{\mathrm{e}, \mathrm{h}}(t)=Q_{\mathrm{e}, \mathrm{h}}(0) \exp \left(-t / \tau_{\mathrm{eff}} \mathrm{e}_{\mathrm{h}}\right)$

where $\tau_{\text {eff }}$ eh are the effective trapping times of electrons and holes, respectively. These have been
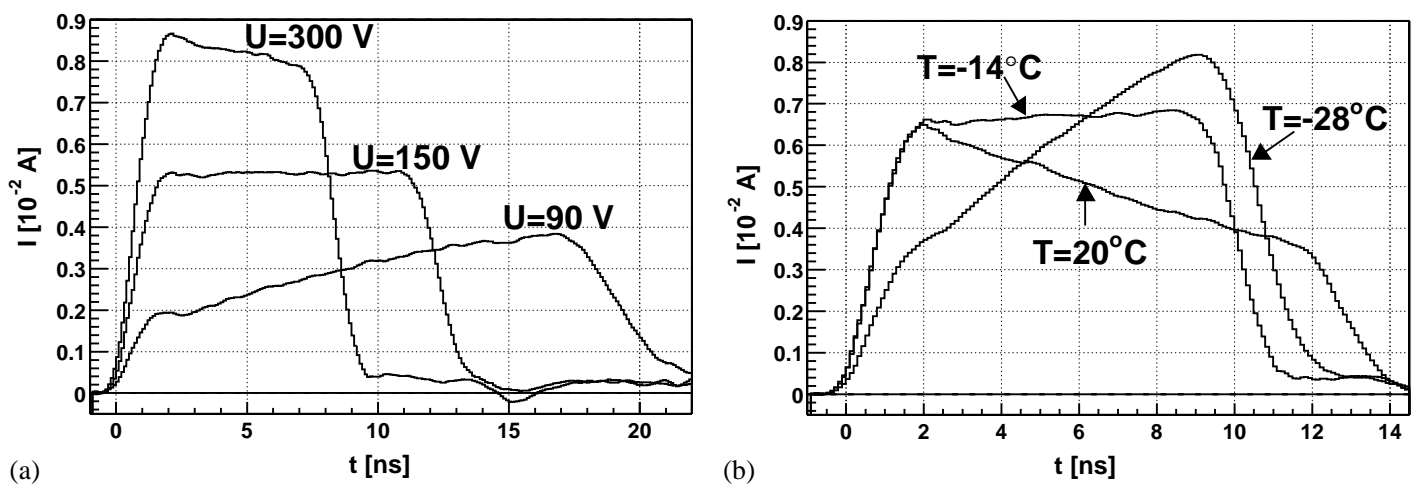

Fig. 2. Observation of induced hole current pulses in the presence of continuous hole generation $\left(\Delta I / S \approx 220 \mu \mathrm{A} / \mathrm{cm}^{2}\right)$ in a sample irradiated to $\Phi_{\text {eq }}=5 \times 10^{13} \mathrm{n} / \mathrm{cm}^{2}$ : (a) hole signals at different bias voltages $\left(T=-10^{\circ} \mathrm{C}\right)$ and (b) hole signals at different temperatures $(V=200 \mathrm{~V})$. 


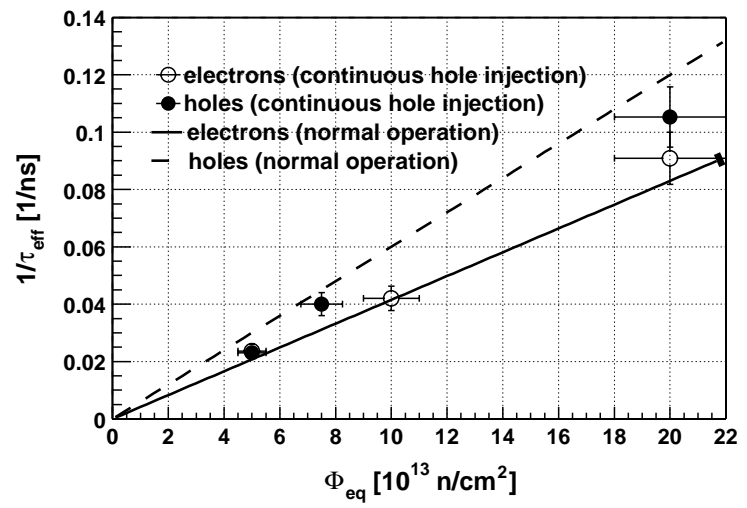

Fig. 3. Effective trapping probabilities in the presence of an enhanced free hole concentration as a function of fluence. The measurements were performed at $T=-10^{\circ} \mathrm{C}$. The free hole concentration was around $p \sim 3.5-5 \times 10^{8} \mathrm{~cm}^{-3}$. A comparison with the values measured in normal diode operation [22] is shown.

recently measured in a systematic way using the charge correction technique [22] but in the absence of continuous hole injection. Since free carriers influence occupation of traps, DC light illumination could affect the trapping times. Therefore, we re-measured the effective trapping probabilities in the presence of an enhanced free hole concentration as a function of irradiation fluence. The results are presented in Fig. 3. The probability of trapping is not significantly influenced by an enhanced free hole concentration of the order of $10^{9} \mathrm{~cm}^{-3}$. Only a slight decrease of trapping probability for holes $1 / \tau_{\text {eff }_{h}}$, and a slight increase of trapping probability for electrons $1 / \tau_{\text {eff }_{e}}$ can be observed in comparison with the normal operation of the detector. These shifts of trapping probabilities are in accordance with an increased number of occupied hole traps and a reduced number of occupied electron traps.

\subsection{Effective space charge}

Having measured the effective trapping times $\tau_{\text {eff }}$ in in Ref. [22], the effective space charge $N_{\text {eff }}$ can be derived from the shape of the TCT signal. In the approximation of a linear field and constant mobility $\mu_{0_{\mathrm{e}, \mathrm{h}}}$, the induced current $I_{\mathrm{e}, \mathrm{h}}$ has an exponential shape in time:

$$
I_{\mathrm{e}, \mathrm{h}}(t) \propto \exp \left[\frac{\mp k t}{\tau_{\mathrm{e}, \mathrm{h}}}-\frac{t}{\tau_{\mathrm{eff}}, \mathrm{h}}\right], \quad t<\text { drift time }
$$

where

$$
\tau_{\mathrm{e}, \mathrm{h}}=\frac{\varepsilon \varepsilon_{0}}{e_{0} \overline{\mu_{0_{\mathrm{e}, \mathrm{h}}}}\left|N_{\mathrm{eff}}\right|} .
$$

$k$ denotes the sign of the space charge and $\mp$ reflects the sign of the drifting charge. $N_{\text {eff }}$ was extracted by fitting the signal shape as given by Eq. (3) to the measured signal with $\tau_{\mathrm{e}, \mathrm{h}}$ as a free parameter. The average mobility from the measured charge collection time $t_{\mathrm{c}}, \overline{\mu_{0_{\mathrm{e}, \mathrm{h}}}}=D^{2} /\left(t_{\mathrm{c}} V\right)$ ( $D$, detector thickness; $V$, bias voltage), was used in Eq. (4). Results of the fit can be seen in Fig. 4. The start of the time interval for the fit was at $t=2 \mathrm{~ns}$ so that the influence of the light pulse duration was small. The end of the fit interval depended on the charge collection time as can be seen in Fig. 4. The upper limit of the bias voltage for which Eq. (3) could be used was imposed by drift velocity saturation which renders Eq. (3) insensitive to electric field and thus $N_{\text {eff }}$. The accuracy of the method was checked by comparing $N_{\text {eff }}$ as obtained from the fit to $N_{\text {eff }}$ calculated from the position of the kink in the charge collection efficiency (CCE) vs. bias voltage curve, and also to $N_{\text {eff }}$ extracted from $C-V$ measurements. An agreement within 10\% between these numbers was found.

In contrast to the minor influence of the enhanced free hole concentration on $1 / \tau_{\mathrm{eff}_{\mathrm{e}, \mathrm{h}}}$, a substantial impact on $N_{\text {eff }}$ was observed. In Fig. 5a, the effective space-charge concentration $N_{\text {eff }}$ is shown as a function of the enhanced free hole concentration at different temperatures. The free hole concentration was varied by changing the bias voltage (see Eq. (1)). The diode had been irradiated to $\Phi_{\text {eq }}=5 \times 10^{13} \mathrm{n} / \mathrm{cm}^{2}$ and the additional current was kept fixed at $\Delta I / S \approx 220 \mu \mathrm{A} / \mathrm{cm}^{2}$. The initially negative $N_{\text {eff }}$ changes to positive as the concentration of holes $p$ grows. The free hole concentration needed to reach the $N_{\text {eff }} \approx 0$ point strongly decreases at lower temperatures. In Fig. 5a, $N_{\text {eff }} \approx 0$ could be reached for temperatures between $T=0^{\circ} \mathrm{C}$ and $10^{\circ} \mathrm{C}$. The applied DC light intensity was too large to operate 

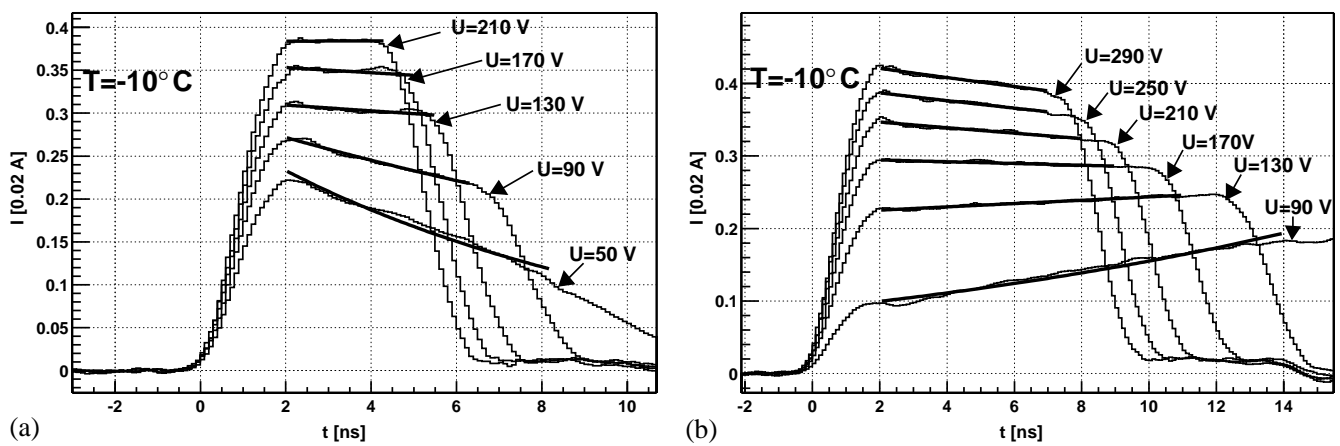

Fig. 4. Results of the fit of Eq. (3) to signals measured at different bias voltages in the presence of an enhanced free hole concentration for: (a) electrons and (b) holes at $T=-10^{\circ} \mathrm{C}$. The diode was irradiated to $5 \times 10^{13} \mathrm{n} / \mathrm{cm}^{2}$.

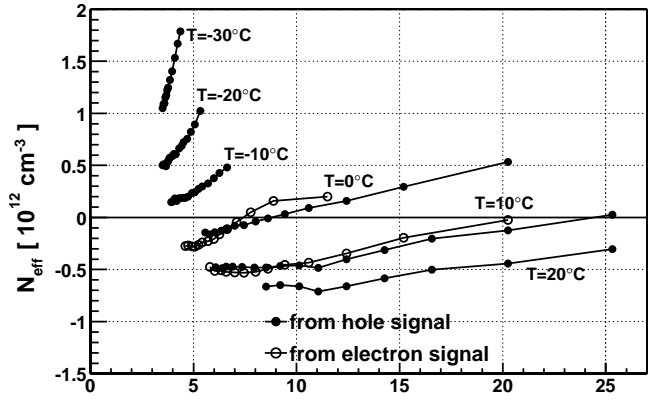

(a)

$\mathrm{p}\left[10^{8} \mathrm{~cm}^{-3}\right]$

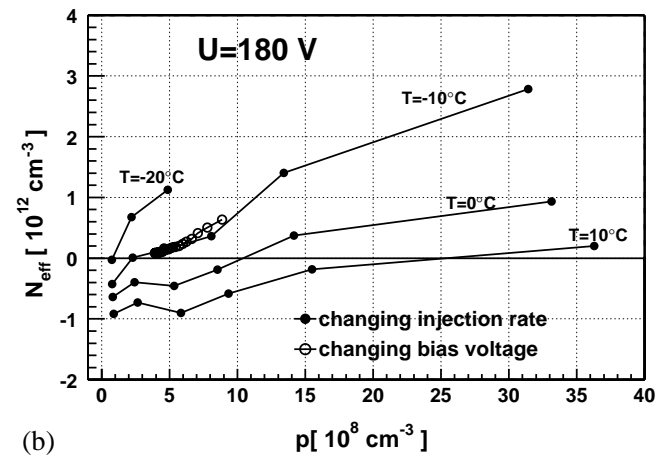

Fig. 5. Effective space-charge dependence on free hole density. (a) Additional current density was $\Delta I / S \approx 220 \mu \mathrm{A} / \mathrm{cm}^{2}$ and the free hole concentration was adjusted by varying the bias voltages. $N_{\text {eff }}$ was measured by fitting either electron or hole signals. The diode had been irradiated to $5 \times 10^{13} \mathrm{n} / \mathrm{cm}^{2}$. (b) Free hole density was regulated by adjusting the continuous light intensity at a constant bias of $180 \mathrm{~V}$. For comparison one measurement obtained with a changing bias voltage at constant $\Delta I / S \approx 200 \mu \mathrm{A} / \mathrm{cm}^{2}$ is also shown. $N_{\text {eff }}$ was measured from the fit to the hole signal. The diode had been irradiated to $7.5 \times 10^{13} \mathrm{n} / \mathrm{cm}^{2}$.

the diode at $N_{\text {eff }} \approx 0$ at $T \leqslant-10^{\circ} \mathrm{C}$ since the free hole concentration could not be decreased sufficiently by voltage increase due to drift velocity saturation. On the other hand, the applied light intensity was not sufficient to reach the $N_{\text {eff }} \approx 0$ point at $T \geqslant 10^{\circ} \mathrm{C}$.

In Fig. 5b, results of measurements on a diode irradiated to $\Phi_{\mathrm{eq}}=7.5 \times 10^{13} \mathrm{n} / \mathrm{cm}^{2}$ are shown. Here, the bias voltage was kept fixed and the DC illumination intensity was regulated. For comparison, and to show the consistency of the method, $N_{\text {eff }}$ obtained with a changing bias voltage at $T=$ $-10^{\circ} \mathrm{C}$ is also exhibited.

At equal temperatures, the points where $N_{\text {eff }} \approx 0$ occur at similar free hole concentrations in both Figs. $5 \mathrm{a}$ and $\mathrm{b}$, although the measurements were performed on two diodes irradiated to different fluences. This striking feature is better exhibited in Fig. 6, where the voltage needed to establish the electric field in the whole detector $\left(V_{\mathrm{FD}}\right)$ vs. temperature is shown at constant additional hole current for diodes irradiated to different fluences. $V_{\mathrm{FD}}$ was measured using the TCT technique as the position of the kink in the collected hole charge vs. bias voltage plots. Collected charge was measured as the integral of the TCT signal over a $60 \mathrm{~ns}$ interval. One can see in Fig. 6 that the difference in $V_{\mathrm{FD}}$ between diodes irradiated to various fluences decreases as the temperature increases. Although a true minimum in $V_{\mathrm{FD}}$ was measured for one diode only, the values of $V_{\mathrm{FD}}$ of other diodes also tend to converge to the same point. The minimum of $V_{\mathrm{FD}}$ is associated with the minimal space charge, i.e., with the point where $N_{\text {eff }} \approx 0$. 


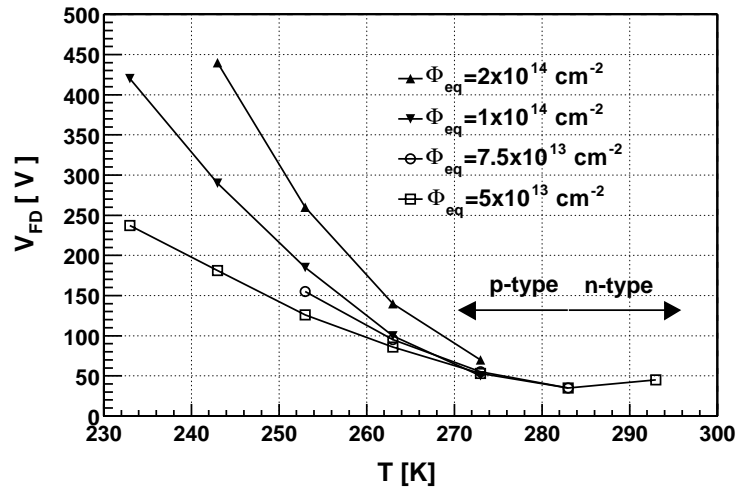

Fig. 6. Dependence of $V_{\mathrm{FD}}$ on temperature at different fluences. The additional hole current density was $\Delta I / S \approx 220 \mu \mathrm{A} / \mathrm{cm}^{2}$.

The fact that at a given temperature and free hole concentration the point where $N_{\text {eff }} \approx 0$ exhibits no dependence on fluence can be explained by the following consideration. In the presence of deep levels and continuous hole injection, $N_{\text {eff }}$ is given by

$$
\begin{aligned}
N_{\text {eff }}= & \Phi_{\text {eq }}\left[\sum_{\text {donors }} g_{t}^{\mathrm{d}} f_{t}^{\mathrm{d}}(t)\left(1-P_{t}^{\mathrm{d}}(p, T)\right)\right. \\
& \left.-\sum_{\text {acceptors }} g_{t}^{\mathrm{a}} f_{t}^{\mathrm{a}}(t) P_{t}^{\mathrm{a}}(p, T)\right] \\
& +N_{\mathrm{D}}\left(\Phi_{\mathrm{eq}}\right)-N_{\mathrm{A}}\left(\Phi_{\mathrm{eq}}\right)
\end{aligned}
$$

where $g_{t}$ is the introduction rate of defects with deep levels, $f_{t}(t) \in[0,1]$ describes the evolution of the defect concentration with time, $P_{\mathrm{t}}(p, T)$ is the occupation probability which for a given trap depends on temperature and free carrier concentration and $N_{\mathrm{D}}\left(\Phi_{\mathrm{eq}}\right)$ and $N_{\mathrm{A}}\left(\Phi_{\text {eq }}\right)$ are the remaining concentrations of initially present shallow dopants. Superscripts $d$ and a denote donor and acceptor defects. Due to the nearly complete removal [1] of the already low initial dopant concentration, the last two terms in Eq. (5) can be neglected. Thus, a cancellation of the two terms in the square bracket yields $N_{\text {eff }} \approx 0$ regardless of the irradiation fluence $\Phi_{\text {eq }}$. The expression in the square brackets can be made to vanish by tuning the occupation probabilities $P_{\mathrm{t}}(p, T)$ by, for example, choosing an optimal temperature $T$ at a given free hole concentration $p$, or vice versa. The occupational probability of a trap with energy $E_{\mathrm{t}}$ in a general stationary state can be written as

$$
\begin{aligned}
& P_{\mathrm{t}}=\left[\frac{c_{p} p+c_{n} n_{\mathrm{i}} \chi_{\mathrm{t}}}{c_{n} n+c_{p} n_{\mathrm{i}} / \chi_{\mathrm{t}}}+1\right]^{-1}, \\
& \chi_{\mathrm{t}}=\exp \left(\frac{E_{\mathrm{t}}-E_{\mathrm{i}}}{k_{\mathrm{B}} T}\right)
\end{aligned}
$$

where $c_{n, p}=v_{\text {th }} \sigma_{n, p}$ are the capture coefficients for electrons and holes, $n_{\mathrm{i}}$ and $E_{\mathrm{i}}$ are the intrinsic carrier density and energy level and $n, p$ are the actual electron and hole densities. For our considerations $n \approx 0$ and $p \approx \Delta p$, the injected hole density. The occupational probability can thus be modified by changing either the temperature or the free hole density.

\subsection{Practical aspects of detector operation in the presence of continuous hole injection}

It was shown in the previous section that by fine-tuning of the DC light intensity and temperature, the effective space charge can be engineered so that $N_{\text {eff }} \approx 0$ even in heavily irradiated detectors. In such a mode, the bias voltage can be set very low, in principle as low as a few volts, and still be sufficient to establish an electric field in the whole thickness of the detector. In practice, a higher voltage is required to reduce the charge collection time and hence limit charge trapping and ballistic deficit. Still, significant reductions of operating bias voltage can be achieved for heavily irradiated detectors. In a detector irradiated to $\Phi_{\text {eq }}=2 \times$ $10^{14} \mathrm{n} / \mathrm{cm}^{2}$ the collected hole charge measured at $200 \mathrm{~V}$ in the presence of continuous hole injection was about the same as the collected hole charge measured at $400 \mathrm{~V}$ in the normal operational mode, both at $T=-10^{\circ} \mathrm{C}$ (see Ref. [17]).

A convenient feature of operating detectors at $N_{\text {eff }} \approx 0$ is that the free hole current needed for compensation of the space charge does not depend on the fluence. The question could arise of the time stability of space-charge cancellation in Eq. (5), as $f_{t}(t)$ need not be the same for all traps. But measurements, both of $\left|N_{\text {eff }}\right|[1]$ as well as of $\tau_{\text {eff }}{ }_{\text {e.h }}$ development in time [22], exhibit a time interval where both $\left|N_{\text {eff }}\right|$ and $\tau_{\text {eff }}$ en remain roughly 
constant, though reverse annealing and changes in trapping are observed upon very long annealing at elevated temperatures. Scaling these results with an activation energy $E_{\mathrm{a}}=1.31 \mathrm{eV}$ [1] to an anticipated operating temperature of $T=-10^{\circ} \mathrm{C}$ and $20^{\circ} \mathrm{C}$ during a maintenance period of 14 days/year yields stable conditions in terms of $f_{t}(t)$ for more than 10 years. Thus, once the working point is set, the same DC light intensity and bias voltage could be used during the entire detector operation. This fluence independence also facilitates the operation of non-uniformly irradiated detectors.

The continuous illumination operation mode also opens up the possibility to use thicker detectors. Once $N_{\text {eff }} \approx 0$ at a given voltage, the electric field is established in the whole volume even in a very thick detector. Since the number of electron-hole pairs created by minimum ionizing particles in the detector is proportional to the detector thickness, higher signals could be obtained with thicker detectors. The trapping of charge, however, reduces the benefits of using very thick detectors, as can be seen in Fig. 7 where the simulated induced charge of a minimum ionizing particle in a pad detector is shown. The simulation was performed assuming simple planar geometry (weighting field $1 / D$ ). Due to the lack of complete parametrization of effective trapping times under light injection, the effective trapping times were calculated using the results from Ref. [22] and mobilities were parametrized according to Ref.
[21] both at $T=-10^{\circ} \mathrm{C}$. Uniform creation of charge along the track was assumed. The details of the signal simulation are explained in Ref. [23]. The created charge drifted through the detector and the induced current was calculated using Ramo's theorem [24] and integrated over $50 \mathrm{~ns}$. It was assumed that the detector had been set to the optimum point by means of continuous hole injection so that $N_{\text {eff }}$ was set to 0 . One can see from Fig. 7a that at a radiation exposure to $\Phi_{\text {eq }}=$ $10^{15} \mathrm{n} / \mathrm{cm}^{2}$ detector thicknesses larger than $400 \mu \mathrm{m}$ do not increase the collected charge significantly. At $\Phi_{\mathrm{eq}}=10^{15} \mathrm{n} / \mathrm{cm}^{2}$ the effective trapping times are of the order of 2 ns [22] and maximum drift times can be estimated by $\tau_{\mathrm{c}}=$ $D / v_{\mathrm{dr}} \approx D^{2} /(V \mu)$, yielding $5 \mathrm{~ns}(V=700 \mathrm{~V})$ to $35 \mathrm{~ns}(100 \mathrm{~V})$ for holes and 1.5-10 ns for electrons in a $400 \mu \mathrm{m}$ thick detector. Now for $\tau_{\mathrm{c}} \gg \tau_{\text {eff }}$, as at the low biases in Fig. 7, the average fraction of collected charge in a pad detector (weighting field $1 / D$ ) is equal to the ratio of the drift path before trapping occurs to the detector thickness:

$$
\begin{aligned}
& Q_{\text {coll }} / Q=v_{\text {dr }} \tau_{\text {eff }} / D \approx \tau_{\text {eff }} V \mu / D^{2}=\tau_{\text {eff }} / \tau_{\mathrm{c}}, \\
& \tau_{\text {eff }} \ll \tau_{\mathrm{c}} .
\end{aligned}
$$

Although the generated charge $Q \propto D$, the collected charge is, as observed in Fig. 7a, smaller for thicker detectors at the same bias. For thicker detectors, the condition $\tau_{\mathrm{c}} \gg \tau_{\text {eff }}$ remains valid to higher biases. The other limiting condition $\tau_{\mathrm{c}} \ll \tau_{\text {eff }}$ (negligible trapping) is not reached even for
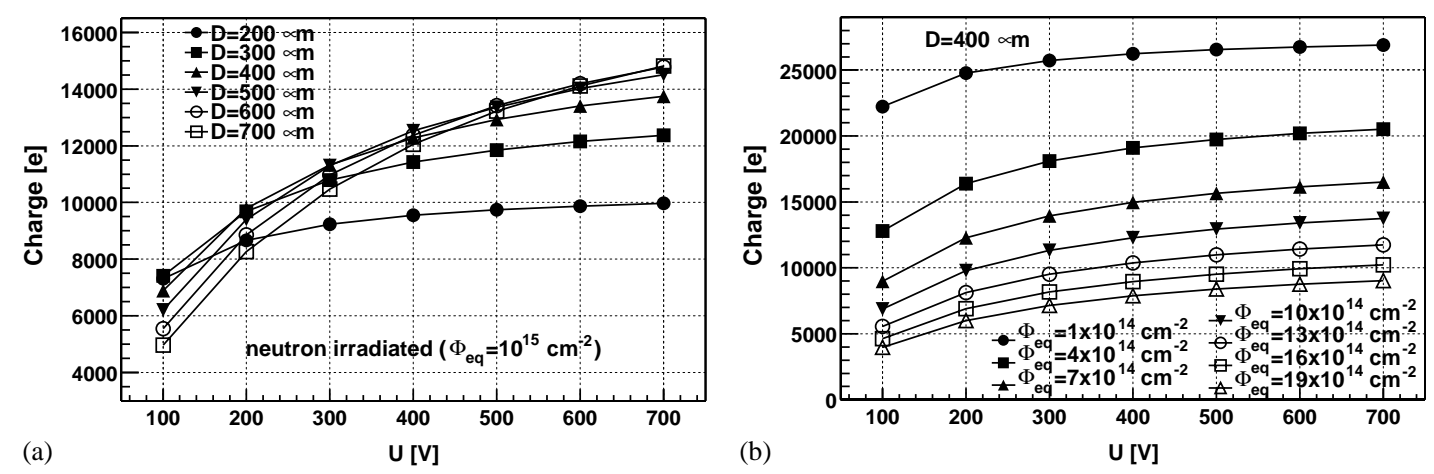

Fig. 7. Simulated most probable collected charge vs. bias voltage with detector at the optimum point $\left(N_{\text {eff }} \approx 0\right.$ ). (a) Detectors of different thicknesses irradiated to $\Phi_{\mathrm{eq}}=10^{15} \mathrm{n} / \mathrm{cm}^{2}$. Note that at this fluence $V_{\mathrm{FD}}$ for a $200 \mu \mathrm{m}$ thick detector in the normal mode of operation is $\approx 700 \mathrm{~V}$. (b) Collected charge for a $400 \mu \mathrm{m}$ thick detector irradiated to different fluences. The most probable charge created by an MIP in silicon is $75 \mathrm{e} / \mu \mathrm{m}$. 
electrons in the thinnest detector at the highest bias. Therefore, the collected charge always stays significantly smaller than the created one. The influence of increasing trapping probability on CCE is clearly exhibited in Fig. 7b for fluences up to $\Phi_{\text {eq }}=1.9 \times 10^{15} \mathrm{n} / \mathrm{cm}^{2}$. In the case of more complex segmented devices like strip detectors, where the bulk of the induced charge comes from the drift of only one carrier type, higher (smaller) CCE than in a pad detector can be achieved if electrons (holes) drift toward the read-out electrodes.

The most important property of detectors is their signal-to-noise ratio. The additional current flowing through the detector contributes to the shot noise. In the case of a silicon detector operated at $T=-10^{\circ} \mathrm{C}$, a free hole density of $p \approx 2 \times 10^{8} \mathrm{~cm}^{-3}$ (see Fig. 5) is needed to reach the $N_{\text {eff }} \approx 0$ point. At a bias of $200 \mathrm{~V}$, the drift velocity of holes is about $3 \times 10^{6} \mathrm{~cm} / \mathrm{s}$ so the estimated additional current density from Eq. (1) is $\Delta I / S \approx 100 \mu \mathrm{A} / \mathrm{cm}^{2}$. Assuming a typical active surface of a strip $S_{\mathrm{s}}=0.03 \mathrm{~cm}^{2}(6 \mathrm{~cm}$ strip at $50 \mu \mathrm{m}$ pitch) or a pixel $S_{\mathrm{s}}=4 \times 10^{-4} \mathrm{~cm}^{2}$ $\left(400 \times 100 \mu \mathrm{m}^{2}\right)$, the resulting increase in the shot noise would be ENC $\sim 900$ e for the strip detector and $\mathrm{ENC} \sim 100 \mathrm{e}$ for the pixel detector, both connected to fast electronics with a peaking time $\tau_{\mathrm{p}}=25 \mathrm{~ns}$ and a simple CR-RC filter [25]. These noise figures are of the same magnitude as the total noise of modern pixel and strip detectors, at such fast shaping times dominated by series (voltage) noise. The two contributions are summed in squares, diminishing to some extent the importance of the additional noise due to DC illumination. This noise increase could be partly compensated by using thicker detectors to obtain larger signals (see Fig. 7), or by operating at lower temperatures where lower free hole concentrations and thus currents are needed to reach the $N_{\text {eff }} \approx 0$ point (see Fig. 5).

The increase of current in the detector caused by continuous light illumination also influences power dissipation. One can compare the leakage current of an irradiated detector, calculated using a damage constant $\alpha=4 \times 10^{-17} \mathrm{~A} / \mathrm{cm}$ [26], with the current increase caused by DC light illumination needed to operate at $N_{\text {eff }} \approx 0$.
At temperature $T=-10^{\circ} \mathrm{C}$ the leakage current density is $I / S \approx 15 \mu \mathrm{A} / \mathrm{cm}^{2}$ at a fluence of $\Phi_{\text {eq }}=$ $2 \times 10^{14} \mathrm{~cm}^{2}$ for a $300 \mu \mathrm{m}$ thick detector. The current increase due to light illumination is $\Delta I / S \approx 100 \mu \mathrm{A} / \mathrm{cm}^{2}$ for the same detector, as estimated above. Although the detectors could be operated at $\approx 200 \mathrm{~V}$ with DC illumination instead of $\approx 500 \mathrm{~V}$ in the normal mode, the power consumption is still a factor of 3 larger than in the normal operational mode. But there the power scales approximately with the square of the fluence (larger $I$ and $V>V_{\mathrm{FD}}$ ), while for DC-illuminated detectors it remains roughly constant, so operation with DC light illumination should prove advantageous even in terms of power consumption at higher fluences.

During the measurements described in this paper the increased density of electrons or holes in the detector was generated by placing the sample in the field of disperse laser light. In practice, this modus operandi can only be used when sufficient place is available behind the detectors. When space is of critical importance other techniques for light distribution, such as modified optical fibers or planar light sources for example in AllnGaP LED technology, can be used.

\section{Conclusions}

It has been demonstrated that continuous hole injection into irradiated silicon detectors changes the electric field profile in a beneficial way. In detectors irradiated beyond the inversion point, space charge in the bulk is negative (p-type). Holes, injected by illumination of the $\mathrm{n}^{+}$detector side with short wavelength light, become trapped into deep levels and compensate the negative space charge. In this mode of operation the electric field can be established through the whole detector at a much lower bias voltage than on normal operation. Adjusting the DC light intensity, bias voltage and/or temperature to tune the free hole concentration and occupation probability of traps, a point of operation can be reached where the initially negative space charge is fully compensated so that $N_{\text {eff }} \approx 0$. This condition is not influenced by irradiation, regardless of the fluence, as soon as the 
remaining initial dopant concentration can be neglected. This fact indicates that the injection of free carriers affects the trap occupation probability, cancelling out the charge of radiation-induced deep donors and acceptors. Thus the dependence on fluence (i.e. number of defects) vanishes. Such behaviour could be exploited to operate detectors irradiated to extreme fluences, where the ultimate limit tends to be given by signal loss due to trapping. The reduction of operational bias voltage in the presence of continuous hole injection opens the possibility of using thicker detectors which give higher signals, although some of the benefit is lost due to charge trapping. The reduction of operating voltage can compensate for the current increase, resulting in an acceptable power consumption. The relatively high current is reflected in a non-negligible increase of shot noise, particularly for strip detectors where the active surface is large. The increase of noise could be compensated in the signal-to-noise ratio by using thicker detectors. Another possibility is operating at lower temperatures where smaller currents are needed to reach the $N_{\text {eff }} \approx 0$ point.

To summarize, operation of silicon detectors with DC hole injection by light illumination opens up a new way of coping with the detector-response deterioration induced by radiation damage. It provides a robust way to extend the radiation tolerance of silicon detectors into the fluence region where operation in the conventional way simply fails. The drawbacks in terms of increased power consumption and noise seem to impose no prohibitive penalty for further developments in this direction.

\section{Acknowledgements}

The authors would like to thank the ROSE collaboration, especially M. Moll, for the STM samples. Special thanks goes to the crew of the TRIGA reactor for their assistance with irradiations.

\section{References}

[1] G. Lindström, et al., Nucl. Instr. and Meth. A 426 (1999) 1.
[2] A. Ruzin, et al., Mater. Sci. Semicond. Process. 3 (2000) 257.

[3] G. Lindström, et al., Nucl. Instr. and Meth. A 465 (2001) 60.

[4] E. Heijne, Low mass semiconductor trackers using thin pixel detectors, Presented at the First Workshop on Radiation Hard Semiconductor Devices for Very High Luminosity Colliders, CERN, 28-30 November 2001.

[5] S.I. Parker, C.J. Kenney, J. Segal, Nucl. Instr. and Meth. A 395 (1997) 328.

[6] V.G. Palmieri, et al., Nucl. Instr. and Meth. A 413 (1998) 475.

[7] E. Fretwurst, et al., Nucl. Instr. and Meth. A 388 (1997) 356.

[8] V. Eremin, Z. Li, I. Iljashenko, Nucl. Instr. and Meth. A 360 (1995) 458.

[9] B. Dezille, et al., Nucl. Instr. and Meth. A 452 (2000) 440.

[10] I. Mandić, et al., TCT measurements at cryogenic temperatures, Presented at the Sixth ROSE Workshop on Radiation Hardening of Silicon Detectors, CERN, 23-24 October 2000, CERN/LEB 2000-006 (307).

[11] C. Da Via, S. Watts, Studies of charge trapping versus temperature: evidence of double junction formation in heavily irradiated samples operated at cryogenic $\mathrm{T}$, Presented at the Sixth ROSE Workshop on Radiation Hardening of Silicon Detectors, CERN, 23-24 October 2000, CERN/LEB 2000-006 (92).

[12] G. Lutz, Nucl. Instr. and Meth. A 377 (1996) 234.

[13] V. Eremin, E. Verbitskaja, Analytical simulation of electric field manipulation and reduction of full depletion voltage by non-equilibrium carrier injection, Presented at RD39 Workshop, unpublished.

[14] B. Dezille, V. Eremin, Z. Li, IEEE Trans. Nucl. Sci. NS-46 (3) (1999) 221.

[15] E. Verbitskaya, et al., IEEE Trans. Nucl. Sci. NS-49 (1) (2002) 258.

[16] K. Borer, et al., Nucl. Instr. and Meth. A 440 (2000) 5.

[17] M. Zavrtanik, et al., IEEE Trans. Nucl. Sci. NS-49 (1) (2002) 264.

[18] E.S. Krištof, Characterization of neutron flux in the exposure channel F19 of the TRIGA Mark II Reactor in Ljubljana, Proceedings of Nuclear Energy in Central Europe'98, Terme Čatež, Slovenia, 7-10 September 1998, p. 43.

[19] D. Žontar, et al., Nucl. Instr. and Meth. A 426 (1999) 51.

[20] C. Canali, G. Ottavian, A. Alberigi, J. Phys. Chem. Solids 32 (1971) 1707.

[21] S. Selberherr, et al., Solid State Electron. 33 (11) (1990) 1425 .

[22] G. Kramberger, et al., Nucl. Instr. and Meth. A 481 (2002) 100.

[23] G. Kramberger, et al., Nucl. Instr. and Meth. A 457 (2001) 550.

[24] S. Ramo, Proc. IRE 27 (1939) 584.

[25] E. Nygärd, et al., Nucl. Instr. and Meth. A 301 (1991) 506.

[26] M. Moll, et al., Nucl. Instr. and Meth. A 426 (1999) 87. 